\title{
Tunka Advanced Instrument for cosmic rays and Gamma Astronomy (TAIGA): Status, results and perspectives
}

L. Kuzmichev ${ }^{1,2, a}$, I. Astapov ${ }^{9}$, P. Bezyazeekov ${ }^{2}$, V. Boreyko ${ }^{10}$, A. Borodin ${ }^{10}$, M. Brückner ${ }^{8}$, N. Budnev ${ }^{2}$, A. Chiavassa $^{4}$, O. Gress ${ }^{2}$, T. Gress ${ }^{2}$, O. Grishin ${ }^{2}$, A. Dyachok ${ }^{2}$, S. Epimakhov ${ }^{1}$, O. Fedorov ${ }^{2}$, A. Gafarov ${ }^{2}$, V. Grebenyuk ${ }^{10}$, A. Grinyuk ${ }^{10}$, A. Haungs ${ }^{14}$, D. Horns ${ }^{6}$, T. Huege ${ }^{14}$, A. Ivanova ${ }^{2}$, D. Jurov ${ }^{2}$, N. Kalmykov ${ }^{1}$, Y. Kazarina ${ }^{2}$, V. Kindin ${ }^{9}$, V. Kiryuhin ${ }^{2}$, R. Kokoulin ${ }^{9}$, K. Kompaniets ${ }^{9}$, E. Korosteleva ${ }^{1}$, D. Kostunin ${ }^{14}$, V. Kozhin ${ }^{1}$, E. Kravchenko ${ }^{11,12}$, M. Kunnas ${ }^{6}$, V. Lenok ${ }^{2}$, B. Lubsandorzhiev ${ }^{3}$, N. Lubsandorzhiev ${ }^{1}$, R. Mirgazov ${ }^{2}$, R. Mirzoyan ${ }^{5,2}$, R. Monkhoev ${ }^{2}$, R. Nachtigal ${ }^{6}$, E. Osipova ${ }^{1}$, A. Pakharukov ${ }^{2}$, M. Panasyuk ${ }^{1}$, L. Pankov², A. Petrukhin ${ }^{9}$, V. Poleschuk ${ }^{2}$, M. Popesku ${ }^{13}$, E. Popova ${ }^{1}$, A. Porelli ${ }^{8}$, E. Postnikov ${ }^{1}$, V. Prosin ${ }^{1}$, V. Ptuskin ${ }^{7}$, A. Pushnin ${ }^{2}$, G. Rubtsov ${ }^{3}$, E. Ryabov ${ }^{2}$, Y. Sagan ${ }^{10}$, V. Samoliga ${ }^{2}$, F.G. Schröder $^{14}$, Yu. Semeney ${ }^{2}$, A. Silaev ${ }^{1}$, A. Silaev(junior) ${ }^{1}$, A. Sidorenko ${ }^{3}$, A. Skurikhin ${ }^{1}$, V. Slunecka ${ }^{10}$, A. Sokolov ${ }^{11,12}$, C. Spiering ${ }^{8}$, L. Sveshnikova ${ }^{1}$, V. Sulakov ${ }^{1}$, V. Tabolenko ${ }^{2}$, B. Tarashansky ${ }^{2}$, A. Tkachenko ${ }^{10}$, L. Tkachev ${ }^{10}$, M. Tluczykont $^{6}$, R. Wischnewski ${ }^{8}$, A. Zagorodnikov ${ }^{2}$, V. Zurbanov ${ }^{2}$, and I. Yashin ${ }^{9}$

1 Skobeltsyn Institute of Nuclear Physics MSU, Moscow, Russia

2 Institute of Applied Physics ISU, Irkutsk, Russia

3 Institute for Nuclear Research of RAN, Moscow, Russia

4 Dipartimento di Fisica Generale Universiteta di Torino and INFN, Torino, Italy

5 Max-Planck-Institute for Physics, Munich, Germany

${ }^{6}$ Institut fur Experimentalphysik, University of Hamburg, Germany

7 IZMIRAN, Moscow, Russia

8 DESY, Zeuthen, Germany

9 NRNU MEPhI, Moscow, Russia

10 JINR, Dubna, Russia

11 Novosibirsk State Univesity, NSU, Novosibirsk, Russia

12 Budker Institute of Nuclear Physics SB RAS, Novosibirsk, Russia

13 ISS, Bucharest, Romania

14 Institut für Kernphysik, Karlsruhe Institute of Technology, KIT, Karlsruhe, Germany

\begin{abstract}
We present the current status of high-energy cosmic-ray physics and gamma-ray astronomy at the Tunka Astrophysical Center (AC). This complex is located in the Tunka Valley, about $50 \mathrm{~km}$ from Lake Baikal. Present efforts are focused on the construction of the first stage of the gamma-ray observatory TAIGA - the TAIGA prototype. TAIGA (Tunka Advanced Instrument for cosmic ray physics and Gamma Astronomy) is designed for the study of gamma rays and charged cosmic rays in the energy range $10^{13} \mathrm{eV}-10^{18} \mathrm{eV}$. The array includes a network of wide angle timing Cherenkov stations (TAIGA-HiSCORE), each with a FOV $=0.6 \mathrm{sr}$, plus up to 16 IACTs $\left(\mathrm{FOV}-10^{\circ} \times 10^{\circ}\right.$ ). This part covers an area of $5 \mathrm{~km}^{2}$. Additional muon detectors (TAIGAMuon), with a total coverage of $2000 \mathrm{~m}^{2}$, are distributed over an area of $1 \mathrm{~km}^{2}$.
\end{abstract}

\section{Introduction}

The progress in understanding the nature of sources of high-energy cosmic rays from our Galaxy and from the Metagalaxy is going along in three directions:

1. The study of secondary gamma quanta, produced by cosmic rays $(\mathrm{CR})$ in the vicinity of the source, where particles are accelerated (experiments H.E.S.S. [1], VERITAS [2], MAGIC [3], MILAGRO [4], HAWC [5], etc.). About 10 sources with gammaray spectra extending up to several tens of $\mathrm{TeV}$ have been discovered. This implies that the parent protons or electrons must have been accelerated to several hundreds of $\mathrm{TeV}$. The measured energy spectra, in general, support the standard model of supernova remnants to be the main sources of Galactic TeV radiation. However, not a single source of gamma rays with energies above $100 \mathrm{TeV}$ has been detected so far.

2. High-energy neutrinos produced by hadronic interactions are studied by neutrino telescopes. Several dozen neutrinos with energies up to a few $\mathrm{PeV}$ have been registered by IceCube [6]. This "breaking the wall" [7] was achieved after four decades of effort and required an array of one cubic kilometer size. Detection of individual astrophysical sources will likely have to wait for future neutrino telescopes (Baikal-GVD [8], KM3NeT [9] and IceCubeGen2 [10]) which are going to be completed in the next 5-15 years.

a e-mail: kuz@dec1.sinp.msu.ru

(c) The Authors, published by EDP Sciences. This is an Open Access article distributed under the terms of the Creative Commons Attribution License 4.0 (http://creativecommons.org/licenses/by/4.0/). 
3. The precise determination of the energy spectrum, mass composition and anisotropy of CRs by detailed measurements of all EAS parameters. In the past, the Tunka Astrophysical Center in the Tunka Valley $(50 \mathrm{~km}$ from Lake Baikal) was solely devoted to this third approach. We will continue the study of CRs with the Cherenkov air-shower array Tunka133 ([11]). This array permits a detailed study of the energy spectrum and mass composition in the energy range from $5 \cdot 10^{15}$ to $10^{18} \mathrm{eV}$. Complementary opportunities to study the mass composition at $10^{17}-10^{18} \mathrm{eV}$ have been opened by the Tunka Radio Extension array (Tunka-REX) [12] and the scintillation array Tunka-Grande [13].

Recently we have also addressed gamma-ray astronomy (i.e. the hitherto most successful way to find individual sources of CRs) and started to deploy a gamma-ray observatory at the Tunka site. The name of this observatory is TAIGA (Tunka Advanced Instrument for cosmic rays and Gamma Astronomy).

In this paper we describe briefly all components of the Tunka Center, present physical results and discuss plans for future extensions.

\section{The EAS array complex for CR studies at $E \geq 10^{15} \mathrm{eV}$}

At present, three arrays to study charged CRs operate at the Tunka site: Tunka-133, Tunka-REX and Tunka-Grande (Fig. 1). Their measurement of the energy spectrum and mass composition is important in order to understand the acceleration limit of the Galactic CR sources and the transition from Galactic to extragalactic CR.

\subsection{Tunka-133}

Tunka-133 consists of 175 optical detectors on the basis of PMTs of type EMI 9350, with a hemispherical photocathode of $20 \mathrm{~cm}$ diameter.

The detectors are grouped into 25 clusters, each with 7 detectors-six hexagonally arranged detectors and one in the center. The distance between the detectors in the cluster is $85 \mathrm{~m}$. 19 clusters are installed in an inner circle of $500 \mathrm{~m}$ radius ("inside" clusters), 6 clusters are placed at a distance of 700-1000 $\mathrm{m}$ from the center ("outside" clusters).

An optical detector consists of a $50 \mathrm{~cm}$ diameter metallic cylinder, containing the PMT. The PMT output pulses are sent via $95 \mathrm{~m}$ coaxial cable RG58 to the center of a cluster and digitized there. The dynamic range of the amplitude measurement is about $3 \cdot 10^{4}$. This is achieved by means of two channels for each detector extracting the signals from the anode and an intermediate dynode of the PMT with different additional amplification factors.

The cluster electronics includes the cluster controller, four 4-channel FADC boards, an adapter unit for connection with the optical detectors and a special temperature controller. The 12 bit and $200 \mathrm{MHz}$ sampling FADC board is based on AD9430 fast ADCs. Station

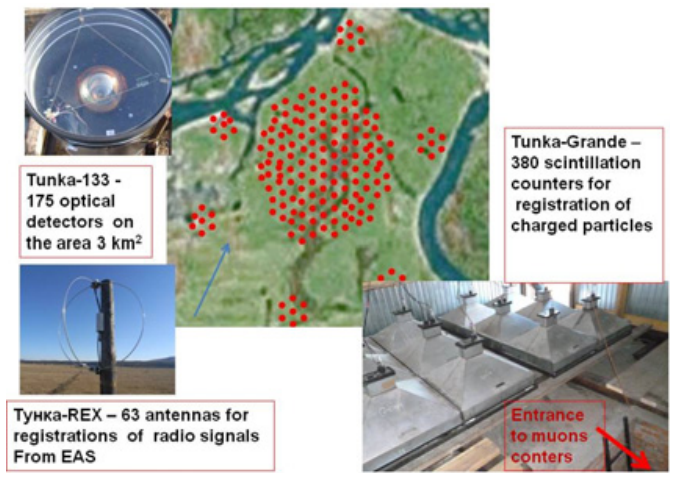

Figure 1. The EAS array complex for study of cosmic rays with $E \geq 10^{15} \mathrm{eV}$ at Tunka Valley.

synchronization and data transmission between DAQcenter and the clusters is realized via optical cables.

\subsection{Tunka-REX}

Detection of air shower radio emission is another technique that can be used to measure EAS parameters. The radio signal is sensitive to the shower energy and the depth of the shower maximum $\mathrm{X}_{\max }$. It is mainly due to the geomagnetic deflection of relativistic electrons and positrons in the shower inducing a time-variable current.

Tunka-REX consists of 63 radio antennae spread over an area of $3 \mathrm{~km}^{2}, 57$ antennae are placed in an inner circle of $500 \mathrm{~m}$ radius. Antennae are connected to the FADC boards in the Tunka-133 clusters and in the Tunka-Grande stations. Readout of the radio data is initialized by the local trigger of the Tunka-133 cluster or scintillation stations and performed via their DAQ systems.

There is a clear correlation between the energy reconstructed from Tunka-REX data and the energy measured by Tunka-133. The Tunka-REX energy precision is about $15 \%$.

A more detailed description of Tunka-REX construction and results is given in [14].

\subsection{Tunka-Grande}

The Tunka-Grande array consists of 19 scintillation stations, with each of the stations having a surface and an underground part. The surface part includes 12 scintillation counters with size $80 \times 80 \times 4 \mathrm{~cm}^{3}$ which formerly operated as part of the EAS-TOP and KASCADE-Grande arrays. There are 8 counters of the same type located underground and operated as muon detectors. The Tunka-Grande DAQ and its synchronization and control systems are practically the same as those of the Tunka-133 array. Each station is operated independently under the station local trigger. Counters of the ground placed counters of the station are divided into two groups, with 6 counters in each. The condition for a station local trigger formation is the presence of a signal from one relativistic particle in each of the two groups in a 100 ns interval.

A description of MC simulation and data processing is presented in [15]. The EAS parameters reconstructed by the Tunka-Grande data are in good agreement with those reconstructed by the Tunka-133 data (Fig. 2). 

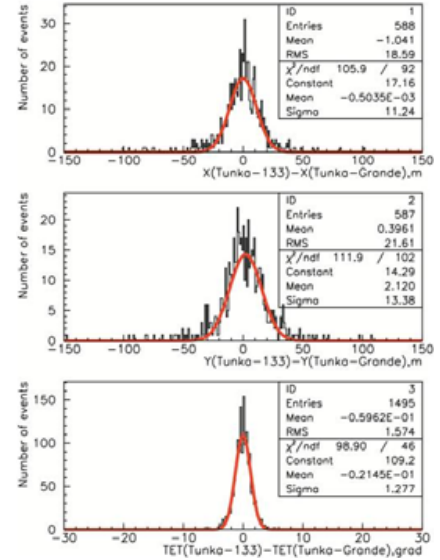

Figure 2. Comparison of the EAS parameters (core position and zenith angle) reconstructed from the data of Tunka-133 and Tunka-Grande.

\section{Short review of the main results}

\subsection{Methods of EAS parameter reconstruction with Cherenkov light}

Our methods of EAS parameter reconstruction have been presented in several papers $([11,16])$. The reconstruction of the EAS core position is performed by fitting measured amplitudes $A_{i}$ with an amplitude distance function (ADF):

$$
A(R)=A(200) \cdot f(R)
$$

The function $f(R)$ is a fit to four different parametrizations according to the distance $R$ (in meters) to the shower core [17]. Four variables in this parametrization $\left(R_{0}, R_{\mathrm{kn}}\right.$, $a$ and $b_{2}$ ), describing the ADF shape in the different ranges of core distance, are related to a single parameter of the ADF shape-the steepness $b_{A}$. The parameter $b_{A}$ is treated as an independent variable during the minimisation procedure. But if two other independent variables define the core position far from the dense parts of the array, $b_{A}$ is treated as a fixed parameter. Its value is derived from the value of $X_{\max }$, obtained from the mean width $\tau_{\text {eff }}$ of the Cherenkov light pulses at a distance of $400 \mathrm{~m}$ to the core. The connection $\tau_{\text {eff }}$ vs. $X_{\max }$ and $b_{A}$ vs. $X_{\max }$ has been obtained and is discussed in [17].

As a measure of energy we use the Cherenkov light flux density at a core distance of $200 \mathrm{~m}-Q(200)$. Reconstruction of $Q(200)$ is made by fitting the measured values of $Q_{i}$ with the lateral distribution function (LDF) [17]. The connection between the EAS energy $\mathrm{E}_{0}$ and $Q(200)$ can be expressed by the following formula:

$$
E_{0}=C \cdot Q(200)^{g}
$$

For an energy range of $10^{16}-10^{18} \mathrm{eV}$, a zenith angle range of $0^{\circ}-45^{\circ}$ and a complex composition, consisting of equal contribution of protons and iron nuclei, CORSIKA simulations provide an index $g=0.94$.

To reconstruct the EAS energy from the Cherenkov light flux one needs to know the absolute sensitivities of the Cherenkov detectors and the atmosphere transparency. To avoid these problems, the method of normalization of the integral energy spectrum to a reference spectrum is used. The reference energy spectrum was measured by the

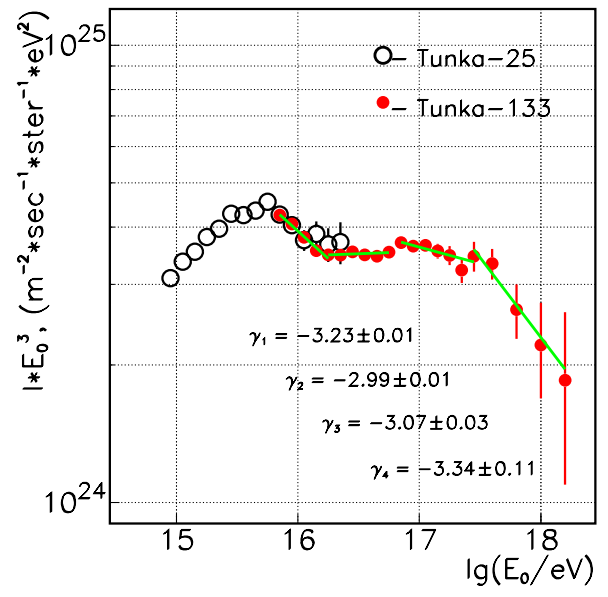

Figure 3. Differential primary cosmic-ray energy spectrum.

QUEST experiment $[18,19]$. The integral energy spectrum obtained for each night of the Tunka-133 operation is normalised to that reference spectrum.

\subsection{All particle energy spectrum}

The Cherenkov light array Tunka-133 operates on clear moonless nights between October and early April. During the rest of the year, the nights are too short and the weather conditions are mostly unsatisfactory. Tunka-133 took data during 5 winter seasons 2009-2010 to 2013-2014. Here we present the data of these 5 seasons. The total time of data acquisition is $1540 \mathrm{hrs}$.

To reconstruct the Tunka-133 spectrum we selected events with zenith angles $\theta \leq 45^{\circ}$ and the core position inside a circle of radius $R_{c} \leq 450 \mathrm{~m}$ for energy $E_{0}<5 \cdot 10^{16} \mathrm{eV}$ and a circle of radius $R_{c}<800 \mathrm{~m}$ for showers with energy $E_{0} \geq 5 \cdot 10^{16} \mathrm{eV}$. Comparison of the spectra for these two effective areas showed that starting from the above mentioned energy, the spectra within the error bars are the same, but the event statistics in the second case is three times larger which is essential for energies $E_{0} \geq 10^{17} \mathrm{eV}$.

The efficiency of shower selection inside the circle with $R_{c} \leq 450 \mathrm{~m}$ reaches $100 \%$ for energies $E_{0} \geq 6 \cdot 10^{15} \mathrm{eV}$. The total number of events above this energy is 270000 . About 3000 events, selected in the circle with $R_{c}<800 \mathrm{~m}$ have $E_{0} \geq 10^{17} \mathrm{eV}$.

The resulting differential energy spectrum is shown in Fig. 3 along with the previous spectrum of Tunka25 [20]. The spectrum of Tunka-133 shows a number of features which indicate deviations from the power law. One can interpret the picture as a much more complicated behaviour than a power law (or multiply broken power law). The power law description can be used only for small parts of the spectrum-not more than half an order of magnitude. At an energy of about $2 \cdot 10^{16} \mathrm{eV}$ the power law index changes from $\gamma=3.23 \pm 0.01$ to $\gamma=2.99 \pm$ 0.01 . This feature was first observed in the KASCADEGrande experiment [21]. Points of the spectrum are consistent with such an index till $E_{0}=5 \cdot 10^{16} \mathrm{eV}$. Above this energy one can notice a single point deflecting from the power law description by about 2 standard deviations. The energy of this point $\left(6.3 \cdot 10^{16} \mathrm{eV}\right)$ coincides with that for a "peak", observed in the GAMMA experiment [22]. 


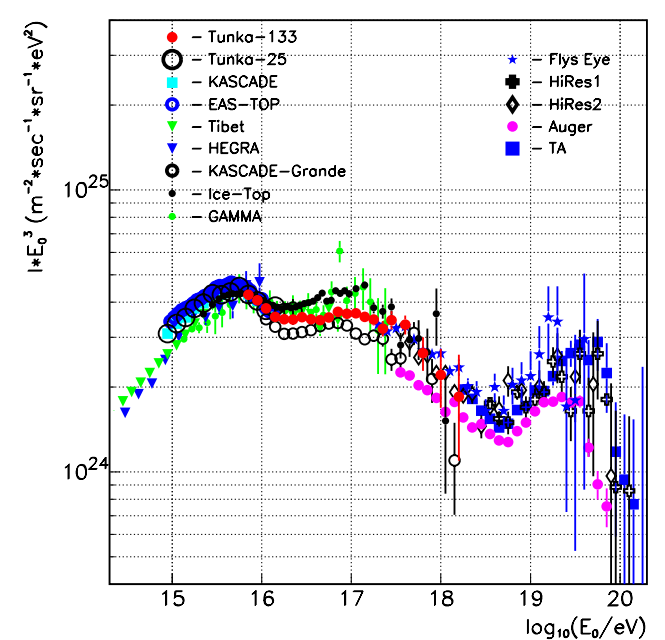

Figure 4. Comparison of energy spectra obtained at Tunka with some other experiments.

Between this point and $E_{0}=3 \cdot 10^{17}$ the index is $\gamma=$ $3.07 \pm 0.03$. The spectrum becomes much steeper with $\gamma=3.34 \pm 0.11$ above the last point (the second "knee").

In Fig. 4 the spectrum is compared with a number of other experimental data. The spectra of all the experiments shown in Fig. 4: KASCADE [23], EAS-TOP [24], Tibet [25], HEGRA [26], are practically indistinguishable at the energy of the first (classical) knee.

In the intermediate energy range $10^{16}-10^{17} \mathrm{eV}$, the Tunka-133 spectrum agrees with those measured by GAMMA [22], KASCADE-Grande [21] and Ice-TOP [27]. Noticeably, in Fig. 4, the difference among the spectra at $E_{0}$ about $10^{17} \mathrm{eV}$ can be eliminated by an energy correction of only $3 \%$. Such an energy shift is much smaller than the absolute accuracy of these experiments.

For the high energy edge, the Tunka-133 spectrum is in agreement with Fly's Eye [28], HiRes [29] and Telescope Array (TA) [30] data, and also (to a lesser extent) with Auger [31].

The fine structure of the spectrum in the range of $10^{15}-10^{17} \mathrm{eV}$ does not contradict the so called rigiditydependent model of the knee origin [32]: any single galactic source accelerates particles up to a limited maximal energy depending on the charge of nucleus $Z$, $E_{\max }(Z)=Z \cdot E_{\max }(Z=1)$ [33]. In this interpretation the position of the first (classical) knee $3 \cdot 10^{15} \mathrm{eV}$ corresponds to the proton cutoff, and the energy $8 \cdot 10^{16} \mathrm{eV}$ indicates the cutoff of iron nuclei $(Z=26)$. The existence of more energetic particles can demand the discussion of new galactic sources of unknown nature or (less probable) extragalactic sources.

\section{From high-energy cosmic rays to multi-TeV gamma-ray astronomy}

For the energy range of gamma quanta above $30 \mathrm{TeV}$ there are a number of fundamental questions which presently have no answers. First of all, there is the question on the sources of Galactic cosmic rays with energies around $1 \mathrm{PeV}$, the energy region approximately adjoining the classical knee in the all-particle energy spectrum. It should be noted that no single photon with an energy of more than $80 \mathrm{TeV}$ has been detected till now.

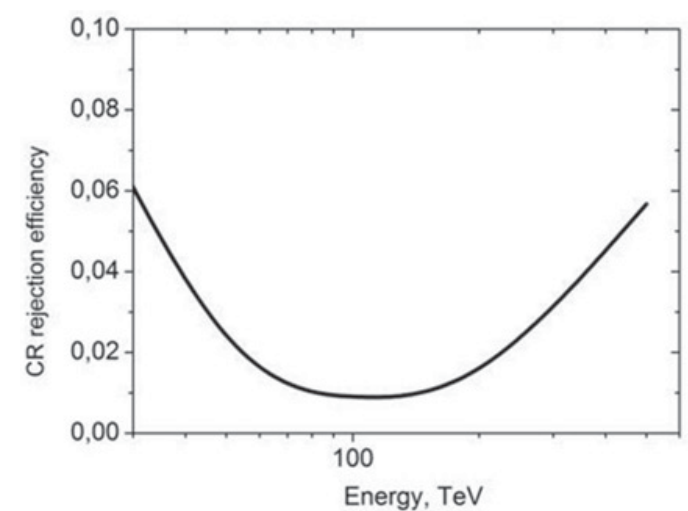

Figure 5. CR rejection efficiency for point-like sources (preliminary).

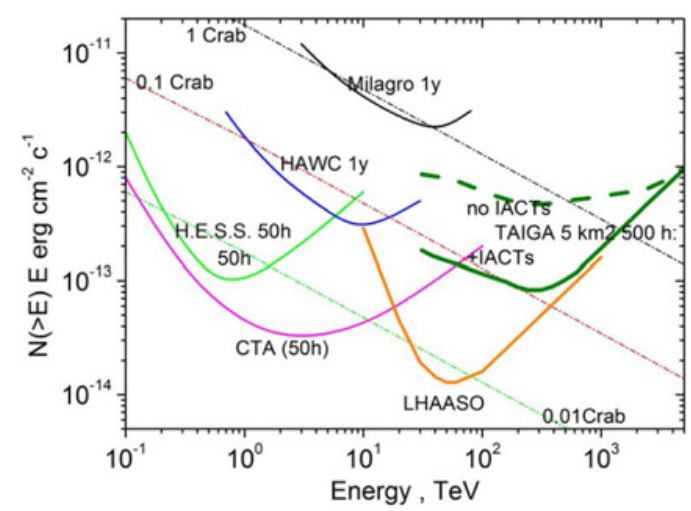

Figure 6. Integral sensitivity for detection of local sources.

\subsection{Concept of the TAIGA experiment}

The gamma-ray Observatory TAIGA is designed to study gamma radiation and the charged cosmic rays in the energy range of $10^{13}-10^{18} \mathrm{eV}$ [34]. The observatory will include a network of wide field of view (0.6 sr) timing Cherenkov light stations, named TAIGA-HiSCORE $[35,36]$ (High Sensitivity Cosmic Origin Explorer), and up to 16 imaging atmospheric Cherenkov telescopes (FOV $10 \times 10$ degrees), covering an area of $5 \mathrm{~km}^{2}$. The capabilities of these Cherenkov arrays are enhanced by muon detectors (TAIGA-Muon) with a total coverage of $2000 \mathrm{~m}^{2}$, distributed over an area of $1 \mathrm{~km}^{2}$.

The advantage of a few IACTs added to the wideangle timing array is their better gamma/hadron separation by image parameter information, while core position, direction and energy can be better reconstructed by the timing array. Combination of information from both components gives a superior result compared to the performance of each single component.

This allows to reduce the CR showers by about 100 times at an energy of $100 \mathrm{TeV}$ for point-like sources, even when the distance between the IACTs is $600 \mathrm{~m}$ (Fig. 5). The detection sensitivity for local sources of a $5 \mathrm{~km}^{2}$ observatory in the energy range of $30-200 \mathrm{TeV}$ is expected to be $10^{-13} \mathrm{erg} \mathrm{cm}^{-2} \mathrm{sec}^{-1}$ for $500 \mathrm{~h}$ of observation or 10 detected events (Fig. 6).

\subsection{The TAIGA prototype-2017}

By the end of 2017, the prototype of TAIGA (Fig. 7) will start operation. The number of detectors (60 stations 


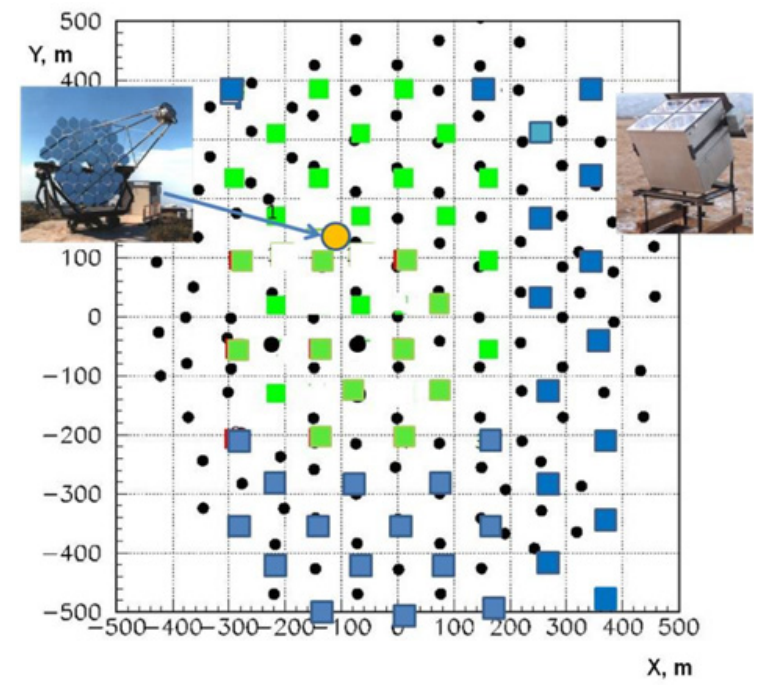

Figure 7. The TAIGA-prototype-2017. Green squares - optical stations of TAIGA-HiSCORE installed in 2014, blue squares optical stations to be installed in 2017. Yellow circle - position of the first TAIGA-IACT. Small black circles - optical detectors of Tunka-133.

of TAIGA-HiSCORE and one IACT) and the area of the prototype $\left(0.6 \mathrm{~km}^{2}\right)$ are approximately $1 / 10$ of the full observatory. The expected integral sensitivity for 200 hours of observation (about 2 seasons of operation) in the range $30-200 \mathrm{TeV}$ is about $10^{-12} \mathrm{erg} \mathrm{cm}^{-2} \mathrm{sec}^{-1}$.

The imaging atmospheric Cherenkov telescope is of Davis-Cotton type with 34 mirrors, $60 \mathrm{~cm}$ diameter each, the focal length is $4.75 \mathrm{~m}$, the camera comprises 560 PMTs of XP1911 type with $2 \mathrm{~cm}$ photocathode diameter. The FOV of the camera is $10^{\circ} \times 10^{\circ}$ [34].

The camera consists of identical clusters, each with 28 PMTs (Fig. 8, left figure). The basis of the cluster electronics is a 64-channel ASIC MAROC-3. Each channel includes a preamplifier with adjustable gain, a charge sensitive amplifier and a comparator with adjustable threshold. This chip has a multiplexed analog output signal which is proportional to the input charge. The chip is connected to a 12-bit external ADC. Signals from each PMT go to 2 channels with gains different by a factor 30 . This results in a full dynamic range of 3000 photoelectrons.

In December 2016 the first TAIGA-IACT was put into operation (Fig. 8, right figure).

Currently, the TAIGA-HiSCORE array is composed of 28 optical stations distributed in a regular grid over a surface area of $0.25 \mathrm{~km}^{2}$ with an inter-station spacing of $106 \mathrm{~m}$ (Fig. 6) [37]. All stations are tilted into the southern direction by $25^{\circ}$ to increase the time for study gamma-quanta fluxes from the first test object-the Crab Nebula. Each optical station contains four large area PMTs with 20 or $25 \mathrm{~cm}$ diameter, namely EMI ET9352KB, or Hamamatsu R5912 and R7081. Each PMT has a Winston cone (made of ten segments of ALANOD 4300UP foil with reflectivity $80 \%$ ) with $0.4 \mathrm{~m}$ diameter and a $30^{\circ}$ viewing angle (field of view is $\sim 0.6 \mathrm{sr}$ ).

The anode signals of all 4 PMTs of the station are summed up. It leads to additional lowering of the energy threshold by a factor of 2 . The minimal distance between stations is $106 \mathrm{~m}$. Each station is connected with the
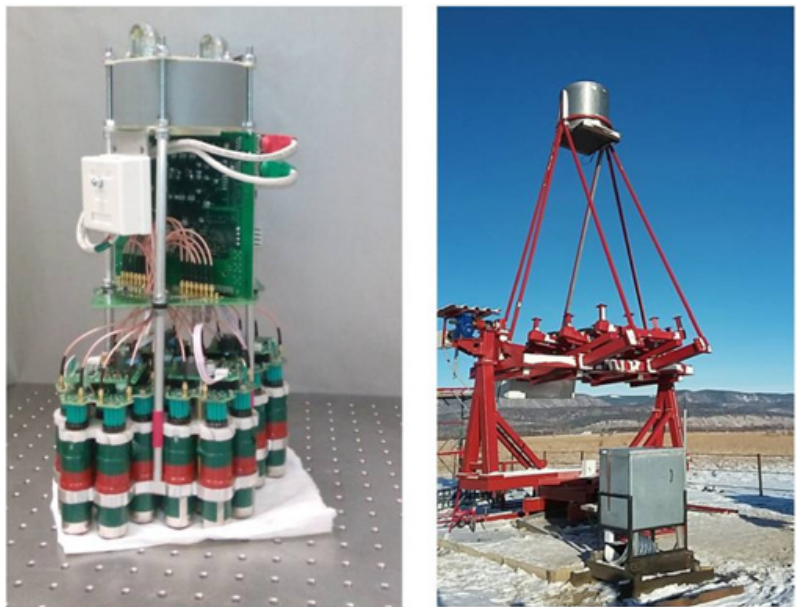

Figure 8. Left figure: Cluster of 28 PMTs. Right figure: The first TAIGA-IACT.

DAQ center by a fiber optic cable for data transfer and synchronization. The synchronization stability of the optical stations reaches about 0.2 ns. Precision calibration is achieved by external light sources. Each station operates independently from the others. The condition for the formation of the local trigger signal is an excess in the sum of anode signals over a threshold level. The threshold is approximately 200 p.e, which corresponds to a Cherenkov light flux of 0.3 photon $\mathrm{cm}^{-2}$ [38]. The counting rate of a single station trigger is about $10-15 \mathrm{~Hz}$. Signals from anode and intermediate dynode are digitized with a step of $0.5 \mathrm{~ns}$ by a special board based on the DRS- 4 chip. A more detailed description of DAQ and synchronization systems is given in [39].

The energy of primary particles is reconstructed by the Cherenkov light flux, Q200, at a distance of $200 \mathrm{~m}$ from the EAS core [16].

Reconstruction of shower parameters was performed using algorithms developed for the Tunka-133 array [11, 16]. Arrival directions of showers are determined by the relative delay of Cherenkov light at each station. In a first step, the arrival direction is reconstructed with a plane wave model of the front. This reconstructed direction is used in the reconstruction of the EAS core. The pulse amplitude is fitted by a parametrization of the amplitude distance function (ADF) [16]. The final EAS arrival direction is reconstructed for the found core position assuming a curved front of the shower.

The accuracy of the reconstruction procedure was checked by MC simulation as well as with experimental data. The latter are presented in $[40,41]$ (chess-board method).

We used two samples of events with zenith angles $28-39^{\circ}$ corresponding to the Crab Nebula when it was in the FOV of TAIGA-HISCORE, and for zenith angles $0-25^{\circ}$. For both samples energies were distributed with a power law energy spectrum with index $\gamma=-2.7$ for protons and helium primaries and index $\gamma=-2.3$ for gamma primaries and energy threshold $30 \mathrm{TeV}$. In simulations the reconstruction method reproduced all the steps of data processing. The accuracy of the arrival direction reconstruction strongly depends on the number of hit stations. The angular resolution is equal to $0.4-0.5^{\circ}$ 


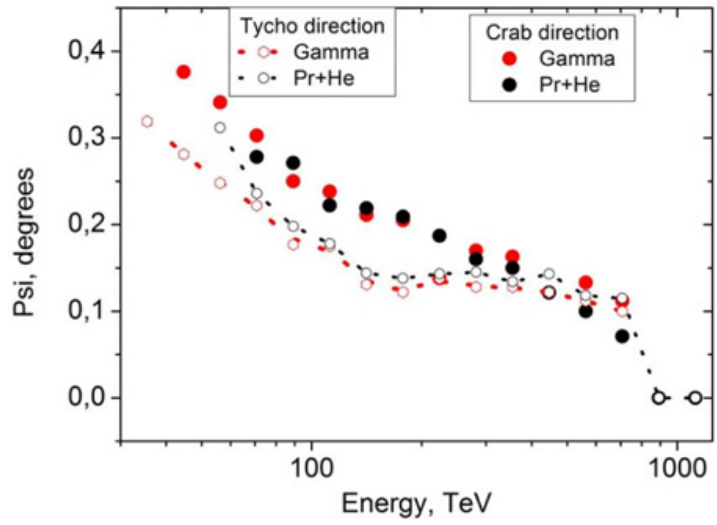

Figure 9. Arrival direction resolution for two samples of events, observed at different zenith angles at the Tunka site: with zenith angles $28-39^{\circ}$ (for the Crab Nebula) and with zenith angles $0-25^{\circ}$ (for the Tycho Nebula). The slope of the energy spectra is equal to $\gamma=-2.3$ for primary gamma rays and $\gamma=-2.7$ for background events $(\mathrm{P}+\mathrm{He})$.

for events with $4-5$ hit stations and about $0.10^{\circ}$ for events with more than 10 hit stations (Fig. 9).

\subsection{First results from TAIGA-HiSCORE}

About 10 million EAS with simultaneous hits in 4 or more stations were recorded during the 35 clean moonless nights of the 2015-2016 winter season. The total time of data acquisition was 210 hours. The energy spectrum obtained by above described procedure is shown in Fig. 10. The energy threshold of almost $100 \%$ efficiency of registration is about $250 \mathrm{TeV}$. Events with zenith angles less than $15^{\circ}$ are used to reconstruct the spectrum below $10^{15} \mathrm{eV}$ and events with zenith angles less than $40^{\circ}$ are used for higher energies.

Our preliminary spectrum is compared with the results of previous experiments in the Tunka Valley, as well as with the results of the direct balloon experiment ATIC-2 and the satellite experiment NUCLEON in Fig. 10. Data taking of the latter experiment is ongoing and one can expect increased statistics by at least a factor of 5 by the end of its operation. Presently the extrapolation of our spectrum to the lower energies does not contradict the results of the direct experiments within their statistical errors.

The Crab Nebula with the pulsar at its center is the most prominent and most studied source in $\mathrm{TeV}$ gammaray astronomy. Therefore it is used as a standard candle for calibration of gamma - ray telescopes. Moreover it is one of a few sources which radiates in the energy range of $10-80 \mathrm{TeV}$, although data spread is still very high. The source can be observed in the field of view of the array from October till March for up to 3.5 hours per night. The maximum full observation time of the source for the described array is about 230 hours per year. The typical observation time is twice less due to bad weather. In the winter of 2015-2016 the achieved observation time was about 60 hours.

The most promising energy range for the gammaray search is the range close to the array threshold. The shower core position for such events is reconstructed as the center of gravity of the pulse amplitudes. The energy is estimated from the average Cherenkov light

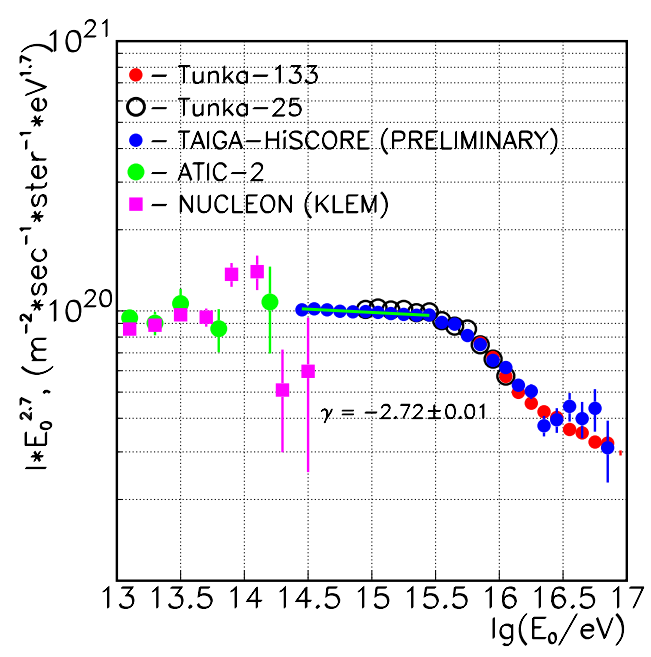

Figure 10. The energy spectrum of primary cosmic rays from the data of the TAIGA-HiSCORE array in comparison with the results of other experiments.

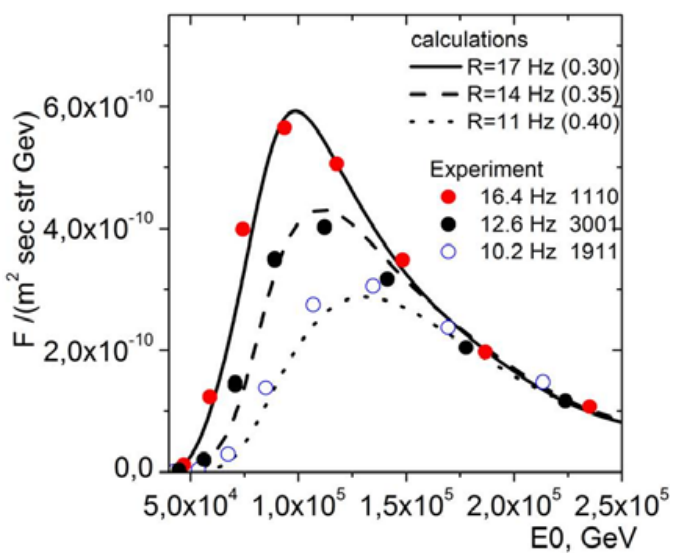

Figure 11. The energy distribution of events in the threshold region.

flux at the four stations closest to the core. The energy distribution for the events close to the array threshold is shown in Fig. 11. The distributions measured in October 2015 and January 2016 are shown separately because the counting rate of the array was different, namely $17 \mathrm{~Hz}$ in October and $10 \mathrm{~Hz}$ in January. The difference of the counting rate is due to different triggering thresholds of the stations. The CORSIKA simulated energy distribution for the different rates is shown in Fig. 11. The peak energies of these distributions change from 100 to $130 \mathrm{TeV}$ in good agreement with the experimentally measured ones. The gamma-ray shower energy is 1.8 times less than that of the charged particle for the same amount of Cherenkov light. So the peak energy of the gamma-ray flux will be in the range of $50-70 \mathrm{TeV}$. At this peak energy the expected number of gamma quanta from the Crab is 10-25 events for 100 hours of observation, depending on the used extrapolation of the spectrum from low energies. The experimental event excess within a $0.4^{\circ}$ bin around the Crab direction is compatible with those expectations.

A first, fast moving pointlike source with TAIGAHiSCORE was detected, while observing the night sky during the 2015/2016 winter-season: a LIDAR operating onboard the International Space Satation (ISS). We found several episodes of $\sim 1 \mathrm{~s}$ duration with extremely high 
array trigger rate (few $\mathrm{kHz}$ as compared to the usual $15 \mathrm{~Hz}$ array rate), with data being compatible with a $4 \mathrm{kHz}$ periodic light source. The reconstructed event directions are coincident with the trajectory of the International Space Station (ISS), when it passes the zenith over the TAIGA site [40]. The strong light source is the CATS-LIDAR [42] onboard the ISS, emitting $\sim 1 \mathrm{~mJ}$ at a wavelength of $532 \mathrm{~nm}$ at $4 \mathrm{kHz}$, and pointing almost vertically downwards. This ISS light source is an interesting object for TAIGA calibration, a detailed analysis is underway.

\subsection{TAIGA-Muon}

We plan to develop a rather cheap and effective detector of muons for the future TAIGA-Muon array [43]. The prototype detector consists of a polystyrene based scintillator in the form of an isosceles triangle. The length of its hypotenuse is $98 \mathrm{~cm}$, the thickness of the plate is $2 \mathrm{~cm}$. Along the legs of the triangle two wavelength shifting bars are arranged. The bars are made from PMMA plastic doped with BBQ dye. The cross section of the bar is $3 \times 17 \mathrm{~mm}^{2}$. The light from the bars is detected by a PMT of type FEU-84 $(\mathrm{QE}=12 \%$ at $500 \mathrm{~nm})$. The reflectors made from PTFE film are placed on the far ends of the bars. The prototype was tested with cosmic ray muons in several points. The mean number of detected photoelectrons from a single muon varies from 9 to 15 . Based on these results we estimate the number of photoelectrons in the future counter to be larger than 20 . This will be achieved by using PMTs with higher quantum efficiency than that of FEU-84 and by optimizing the amount of BBQ dye in the WLS bar with larger cross-section $5 \times 20 \mathrm{~mm}^{2}$. We assume that 20 detected photoelectrons will be enough to ensure $100 \%$ detection efficiency of a cosmic muon and a long lifetime of the detector.

\section{Conclusion}

A unique complex of installations for the study of high-energy cosmic rays is constructed at the Tunka Astrophysical Center. The complex includes the Tunka133 array registering EAS Cherenkov light, TunkaREX for registration of EAS radio emission and the Tunka-Grande array for registration of charged particles (electrons and muons). The joint operation of these arrays opens new opportunities for studying the cosmic radiation with energies more than $10^{16} \mathrm{eV}$.

The first significant steps towards the gamma-ray observatory TAIGA have been done. The first IACT of TAIGA is now in the commissioning stage.

The first season of operation of the TAIGA-HiSCORE prototype as part of the TAIGA prototype with its 28 optical stations was rather successful and demonstrated good performance and high reliability of equipment.

32 additional stations will be deployed in 2017. Before the winter season 2017-2018 the TAIGA configuration will include 60 wide angle stations arranged over an area of $0.6 \mathrm{~km}^{2}$, and one single IACT. The expected integral sensitivity for 200 hours of a source observation (about 2 seasons of operation) in the range $30-200 \mathrm{TeV}$ is about $10^{-12} \mathrm{erg} \mathrm{cm}^{2} \mathrm{sec}^{-1}$.

This work was supported by the grant 15-12-20022 of the Russian Science Foundation, the Russian Federation Ministry of Education and Science (agreement 14.B25.31.0010, zadanie
3.9678.2017/BCh, 3.904.2017/PCh) (part 4), by the Deutsche Forschungsgemeinschaft (DFG) and by the German Helmholtz Association.

\section{References}

[1] A. Abramowski et al. (HESS Colaboration), Nature 531, 476 (2016)

[2] V. Acciari et al. (Veritas Collaboration), Astrophys. J. 730, L20 (2011)

[3] S. Ansoldi et al. (MAGIC Collaboration), Astrn. Astrophys. 585, A133 (2016)

[4] A. A. Abdo et al., Astrophys. J. 700, L127 (2009)

[5] A. Abeysekara et al. (HAWC Collaboration), arXiv: 1701.01778 (2017)

[6] M. Aartsen et al. (IceCube Collaboration), Phys. Rev. Lett. 115, 081102 (2015)

[7] C. Spiering, Proc. 16th Lomonosov Conference on Elementary Particle Physics, Moscow (2013) 105

[8] A. Avrorin et al. (Baikal Collaboration), EPJ Web Conf. 121, 05003 (2016)

[9] S. Adrian-Martinez et al. (KM3NeT Collaboration), J. Phys. G: Nucl. Part. Phys. 43, 084001 (2016)

[10] M. Aartsen et al. (IceCube Collaboration), arXiv: 1510.05228

[11] S. Berezhnev et al. (Tunka Collaboration), NIM A 692, 98 (2012)

[12] P.A. Bezyazeekov et al. (Tunka-REX Collaboration), JCAP 01, 052 (2016)

[13] R. Monhoev et al. (Tunka Collaboration), Bull. Russian Academy of Sciences: Physics 81, 504 (2017)

[14] D. Kostiunin et al. (Tunka-REX Collaboration), these proceedings

[15] N. Budnev et al., Moscow University Physics Bulletin 4, 357 (2014)

[16] S. Berezhnev et al (Tunka Collaboration), NIM A 756, 94 (2014)

[17] Prosin V.V., et al., NIM A 756, 94 (2014)

[18] E. Korosteleva et al., Int.J.Mod Phys. A 20, 6837-6839 (2005)

[19] E.E. Korosteleva et al., Nuclear Physics B (Proc. Supp.) 165, 74-80 (2007)

[20] Budnev N., et al., Astropart. Phys. 50, 18-25 (2013)

[21] Apel W.D., et al. (KASCADE-Gr. Collaboration), Astropart. Phys. 36, 183 (2012)

[22] Garyaka A.P., et al., Journal of Physics G: Nuclear and Particle Physics ID=35, 115201 (2008)

[23] Antony T., et al., Nucl. Instr. and Methods in Physics Research A 513, 490 (2003)

[24] Aglietta M., et al., EAS-TOP Collaboration, Astropart. Phys. 10, 1 (1999)

[25] Amenomori M., et al., (Tibet Col.), Astrophys. J. 678, 1165 (2008)

[26] Arqueros F., et al., The HEGRA Collaboration, Astron. Astrophys. 359, 682 (2000)

[27] Aartsen M.G., et al., Phys. Rev. Lett. D 88, 042004 (2013)

[28] Abu-Zayyad T., et al., Phys. Rev. Lett. 84, 4276 (2000)

[29] Sokolsky P. for the HiRes Collaboration, Nuclear Physics B (Proc. Suppl.) 212-213, 74 (2011)

[30] Abu-Zayyad T., et al., Astropart. Phys. 48, 16 (2013) 
[31] Schulz A. for the Pierre Auger Collaboration, Proc. 33rd ICRC Rio De Janeiro ID=769 (2013)

[32] Sveshnikova L., et al., Nuclear Physics B (Proc. Suppl.) 218-224, 256 (2014)

[33] Erlykin A., Wolfendale A., Martirosov R., CERNCourier 21, 201 (2011)

[34] N. Budnev et al. (TAIGA Collaboration), Journal of Physics: Conference Series 718, 052006 (2016)

[35] M. Tluczykont et al., Adv. Space Res. 48, 1935-1941 (2011)

[36] M. Tluczykont et al., Astropaticle Phys. 56, 42 (2014)

[37] I.Yashin et al (TAIGA Collaboration), ICPPA-2015, J. of Physics: Conference Series 675, 032037 (2015)
[38] S.F. Berezhnev et al. (TAIGA Collaboration), Bull. Russ. Acad. Sci. Phys. 79, 38 (2015)

[39] Gress O. et al. (TAIGA Collaboration), NIM A 845, 367 (2017)

[40] M.Tluczykont et al (TAIGA Collaboration), Proceedings of RICAP16 6th Roma International Conference on Astroparticle Physics. EPJ Web Conferences

[41] A.Porelli et al. (TAIGA Collaboration) Proceeding of ECRS 2014, J.of Phys: Conf.Ser. 632, 012041 (2015)

[42] http://cats.gsfc.nasa.gov/

[43] Budnev N. et al. (TAIGA Collaboration), NIM A 845, 384 (2017) 